\title{
Pilomatrix carcinoma on the left side of the parotid region: A case report and review of the literature
}

\author{
JI-FENG LIU ${ }^{1}$, BIN LI $^{2}$, ZI-XUAN FAN ${ }^{3}$, TING JIAO $^{4}$, CHAO LI $^{1}$, \\ SHENG QIN ${ }^{5}$, JIN-YI LANG ${ }^{3}$ and JIN-XIU CHEN ${ }^{6}$ \\ ${ }^{1}$ Department of Head and Neck Surgery, Sichuan Cancer Hospital; ${ }^{2}$ Department of Otolaryngology, \\ Sichuan Provincial People's Hospital; ${ }^{3}$ Department of Radiation Oncology, Sichuan Cancer Hospital, \\ Chengdu, Sichuan 610041; ${ }^{4}$ North Sichuan Medical College, Nanchong, Sichuan 637007; \\ Departments of ${ }^{5}$ Pathology and ${ }^{6}$ Imageology, Sichuan Cancer Hospital, Chengdu, Sichuan 610041, P.R. China
}

Received September 9, 2014; Accepted April 15, 2015

DOI: $10.3892 / 01.2015 .3192$

\begin{abstract}
Pilomatrix carcinoma (PC) is a rare neoplasm, particularly in the parotid region. Thus, it is easily misdiagnosed and an optimal treatment regimen has not yet been established. The present study reports the case of a 43 -year-old female who presented with a PC of the parotid region and reviews the associated published literature. The patient underwent three surgical excisions prior to the tumor being completely removed, and was misdiagnosed four times prior to the correct diagnosis. Once the tumor was completely removed, the patient received radiation therapy (RT). At the 2-year follow-up, the patient remained free of local recurrence and metastasis. To the best of our knowledge, only 3 cases of $\mathrm{PC}$ on the parotid region have been reported. Although an optimal treatment regimen has not been established, surgery with wide margins is recommended, with RT and chemotherapy producing mixed results.
\end{abstract}

\section{Introduction}

Pilomatrix carcinoma (PC) is a rare and malignant adnexal tumor of hair matrix origin (1). The tumor is a dermo-hypodermic in nature, with a low metastatic potential, but a high risk of recurrence following excision. PC is characterized by a locally-aggressive and low-potential malignant lesion, which is likely to be misdiagnosed. However, distant metastasis and mortality have also been reported (2). To highlight a further case of this extremely rare occurrence, the present study reports a case of PC of the parotid region

Correspondence to: Dr Chao Li, Department of Head and Neck Surgery, Sichuan Cancer Hospital, 55 Renmin South Road 4th Section, Chengdu, Sichuan 610041, P.R. China

E-mail: headneck@qq.com.cn

Key words: pilomatrix carcinoma, parotid, surgery, radiation therapy, chemotherapy that was misdiagnosed numerous times, and reviews the pertinent literature.

\section{Case report}

A 43-year-old female first visited the Department of Head and Neck Surgery of Jiangyou City People's Hospital (Jiangyou, Sichuan, China) in 2009 due to a tenacious subcutaneous nodule that was $1.0 \mathrm{~cm}$ in diameter on the left side of the parotid region. The small, subcutaneous nodule was asymptomatic, and had been slowly increasing in size for the past 5 years. The nodule was surgically excised under local anesthesia with a false clinical diagnosis of an adenolymphoma of the parotid gland. However, 1 year later, a tenacious mass with an unclear boundary reappeared at the previous site. A secondary excision was performed at the same hospital, and the histological evaluation revealed a squamous epithelial cell mass and cells with hyperchromatic nuclei among fibrous tissue, reactive hyperplasia of the lymph node and partial epithelial proliferation among the tissues. A gray-black nodule with swelling and effusion appeared 3 months later at the site of the primary lesion. The patient was admitted to the same hospital once more. A biopsy of the nodes revealed a well-differentiated squamous cancer. The patient was then transferred to our hospital for further diagnosis and therapy.

Upon physical examination, a $2.0 \times 2.0-\mathrm{cm}$, tender, firm, non-fluctuant, gray-black lesion was noted on the skin surface of the left side of the parotid region (Fig. 1). There was no palpable lymphadenopathy of the left side of the neck. Sensation around the area and salivation were normal. There was no facial nerve dysfunction or detectable adenopathy. The remainder of the physical examination was otherwise unremarkable, as well as the results of routine laboratory tests and chest X-rays. Computed tomography revealed a solid mass measuring $2.0 \times 2.1 \times 1.5 \mathrm{~cm}$, which infiltrated the left parotid gland (Fig. 2). As the exact nature of the lesion was not certain, frozen sections were analyzed: Microscopic examination revealed that the tumor was composed of sheets and nests of basaloid cells with prominent central necroses and nuclear pleomorphism, and a diagnosis of cancer, likely to be a pigmented basal cell carcinoma, was determined. The selected 


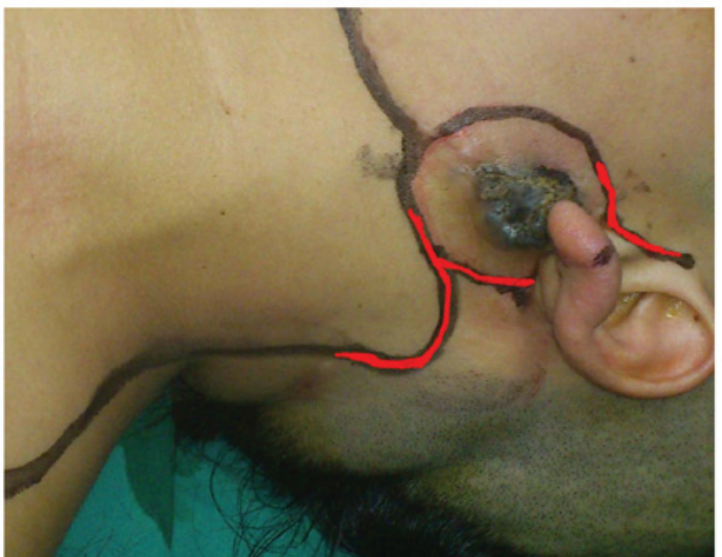

Figure 1. Pilomatrix carcinoma occurring on the skin surface of the left side of the parotid region as a $2.0 \times 2.0-\mathrm{cm}$, tender, firm, non-fluctuant, gray-black lesion. The black lines indicate the incision lines and the safe margin. The red lines shows the bilobed flap.

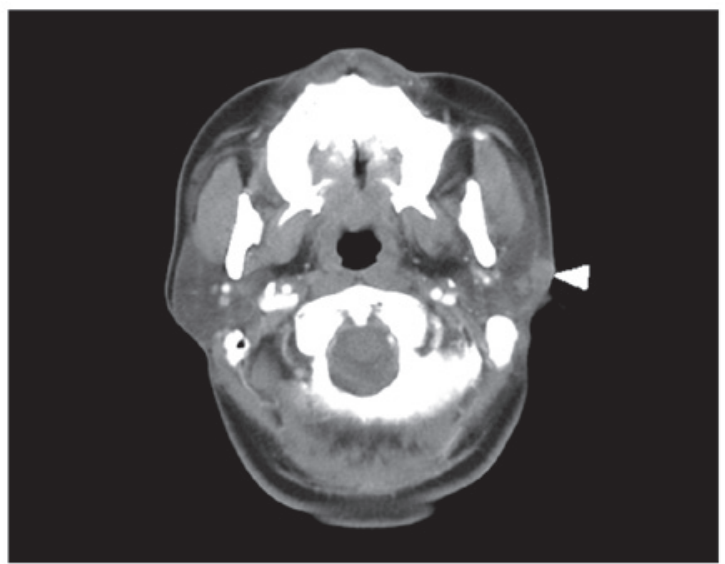

Figure 2. Computed tomography image showing a solid, obscure boundary mass infiltrating the left parotid gland (arrowhead).

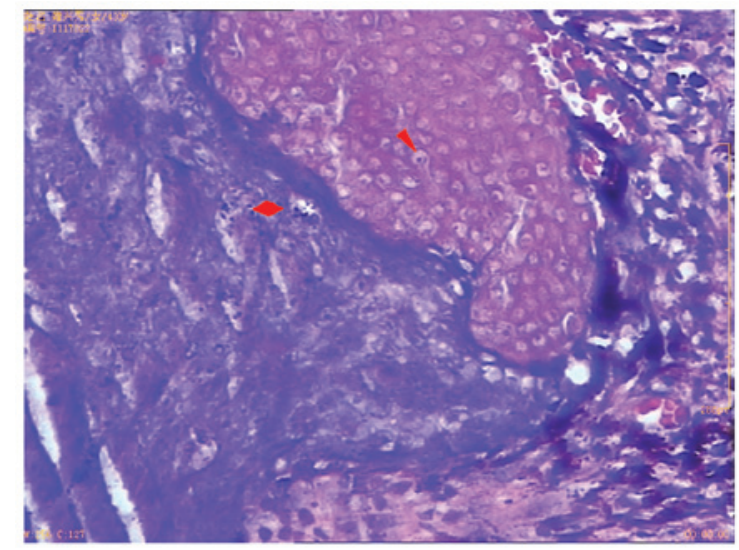

Figure 3. Basaloid cells with pleomorphic nuclei, prominent nucleoli and frequent atypical mitoses (red diamond), and clear spaces reminiscent of shadow cells (red arrowhead). Hematoxylin and eosin stain; magnification, $x 400$.

treatment strategy consisted of resection of the whole tumor together with the left parotid gland, submaxillary salivary gland and cervical lymph nodes of the left side of the neck. The

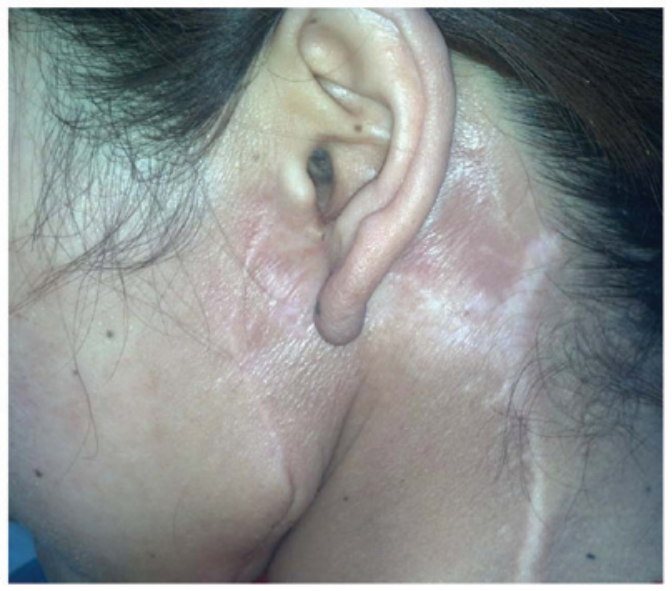

Figure 4. Cosmetic outcome 14 months post-operatively demonstrating excellent wound healing.

tumor was located subcutaneously, reaching the parotid fascia, buccal branches and ramus marginalis mandibulae nervi facialis. After confirming a complete resection histologically, a bilobed flap reconstruction was immediately performed to cover the resection area (Fig. 1). The resected tumor was submitted for pathological examination. Microscopic examination revealed that the tumor was composed of sheets and nests of basaloid cells with prominent central necrosis and nuclear pleomorphism, and numerous shadow cells in the necrotic area (Fig. 3). A diagnosis of PC was therefore formed. The patient experienced an uneventful post-operative course and excellent wound healing, with nearly symmetrical facial nerve function. Due to the previous recurrences following multiple surgeries, the patient received fractionated external beam radiation therapy (RT) to the left parotid region and neck at a total dose of 50 Gy in 25 fractions. Short-term follow-up, including clinical and ultrasound examinations, was performed for the early detection of possible tumor recurrence at 12-week intervals. At the 2-year post-RT follow-up, a routine pathological and ultrasound examination demonstrated that the patient remained free of local recurrence and metastasis (Fig. 4).

The protocol for the present retrospective study was approved by the Ethics Committee of Sichuan Cancer Hospital, and written informed consent was obtained from the study patient.

\section{Discussion}

Since PC was initially described by Lopansri and Mihm in 1980, only 136 cases of PC (including 15 cases published in Chinese with English abstracts) have been reported in the English literature worldwide (3-5). Another 28 such cases have been reported in the Chinese literature. To the best of our knowledge, only a few cases have been reported in the parotid region (6-8). Although $\mathrm{PC}$ occurs more commonly in males than in females (ratio, 3:1) in the published English literature excluding Chinese cases, the rate is approximately 1:1 (22:23) in the Chinese cases (including the present case). The patients in these previously reported cases range in age from 14 months to 93 years, with a mean of 46.3 years (9). The most common site of PC is the head and neck, occurring in $60 \%$ of patients, 
followed by the upper extremities, trunk and lower extremities (10).

PC manifests clinically as a multicolored, asymptomatic cystic or solid mass. On presentation, PC varies in size from 0.5-20.0 cm (average, $3.6 \mathrm{~cm}$; median, $2.5 \mathrm{~cm}$ ) and the lesion can be present from several months to years prior to treatment. Due to a lack of clear histological criteria to distinguish PC from other matrical tumors, the diagnosis of this neoplasm can be challenging. PC tumors consist of pleomorphic basaloid cells with prominent nucleoli and frequent mitoses (a number of which are atypical) accompanied by central areas with keratotic material, shadow cells and foci of necrosis (11). The transition to squamous cells, clear cells, areas of necrosis and frequent mitoses is associated with metrical cornification in PC (3). Additional characteristics include the appearance of an infiltrative pattern that is asymmetric with frequent invasion of fat and other underlying structures, such as vascular, lymphatic or perineural invasion $(3,12)$. In the present case, sheets and nests of basaloid cells with prominent central necroses and nuclear pleomorphism, typical shadow cells, atypical mitoses and infiltration of the parotid gland and nervi facialis were observed.

The differential histological diagnosis is necessary to exclude other tumors derived from hair follicles, including proliferating pilar cysts, basal cell carcinoma with metrical differentiation, pilomatrixoma, trichoepithelioma, squamous cell carcinoma, lymphoepithelioma-like carcinoma of the skin and mixed tumors of the skin $(13,14)$. A proliferating trichilemmal cyst comprises lobules or sheets of squamous nests with trichilemmal keratinization, however, ghost cells or basaloid cells are absent in these lesions (14). Proliferating pilomatrixoma and basal cell carcinoma with matrical differentiation may resemble PC clinically and histologically. However, PC can be distinguished from these tumors by the appearance of sheets and islands of basaloid matrical cells with considerable nuclear pleomorphism and an asymmetric infiltrative pattern, with frequent invasion of fat and other underlying structures (12). Trichoepitheliomas consist of islands of basaloid cells, which may contain shadow cells and keratin horn cysts. However, characteristics of malignancy, including infiltrative growth, necrosis, prominent nucleoli and pleomorphism, are absent in these tumors (14). Squamous cell carcinoma may contain cytological features similar to the basaloid cells of PC, but lack ghost cells (15). Lymphoepithelioma-like carcinoma contains epithelial cell islands that may resemble PC. However, the tumors lack shadow cells or squamous differentiation, while PC lacks a prominent lymphoplasmacytic infiltrate (14). Shadow cells in large horn cysts may be present in mixed tumors of the skin, however, characteristics of malignancy, including infiltrative growth, necrosis, prominent nucleoli and pleomorphism, are absent (15). Due to the rarity of PC, the difficulty of diagnosis and the lack of experience in such cases, the present case was misdiagnosed numerous times. As this tumor is inclined to arise in the head and neck region, particularly the parotid region, the rare occurrence and atypical presentation of such tumors pose a diagnostic dilemma, often resulting in a misdiagnosis of primary parotid neoplasm. In the present case, the PC was misdiagnosed as adenolymphoma. Diagnostic imaging may be of use in the clinical setting in order to clearly demonstrate the association between the mass and the parotid parenchyma (16), prior to parotid infiltration.
The ability of PC for infiltration both laterally and in depth is significant. Local relapses are frequent, as observed in the present case. PC can disseminate via the lymphatic or the blood system, and forms metastases. As PC is a rare entity, the standards of surgical management are not well-defined. Therefore, the preferred treatment method involves excision of the tumor with wide safety margins. Previous studies have recorded margins ranging from $0.5-3.0 \mathrm{~cm}$ where feasible $(14,15,17)$. In the present case, a complete tumor resection was performed using a wide local resection with clear margins $(1.0-2.0 \mathrm{~cm})$, and the larger defect was reconstructed by a bilobed flap. With regard to RT, only a limited number of cases employing the treatment have been described and its role in the therapy for PC remains unclear, mainly due to the lack of experience and diverse results reported following its use (18). Tselis et al summarized 6 cases that had been treated initially with RT or with RT following surgery, and observed no local recurrence after 2 years of follow-up (18). The present patient was administered a total RT dose of $50 \mathrm{~Gy}$ as a post-operative supplementary treatment, and has remained free of local recurrence and metastasis for 2 years. We suggest that RT may therefore be an effective method for the treatment of PC. As a result of prior therapy, the tumor bed is generally more hypoxic with recurrent lesions. The higher radiation doses that are applied with interstitial high dose rate brachytherapy may be of advantage in treating not only recurrent $\mathrm{PC}$, but also residual or newly diagnosed PC (18). Certain authors consider that chemotherapy has not been proven effective $(1,3)$. However, to the best of our knowledge, systemic chemotherapy has been used in 2 cases of metastatic PC, and these cases have exhibited a response $(1,19)$.

In conclusion, $\mathrm{PC}$ is a rare malignant tumor that is easily misdiagnosed. The histological diagnosis, based on several factors (shadow cells, necrosis, nuclear atypia, infiltration and abnormal mitosis) is difficult to prove. An optimal treatment regimen has not been established. A surgical procedure with wide margins is recommended to avoid recurrence when the staging shows no metastasis. RT has provided mixed results and has been most commonly used as an adjuvant therapy for PC. Chemotherapy is generally considered to be ineffective, although 2 cases of metastatic PC have shown a response.

\section{Acknowledgements}

The study was supported by grants from the Sichuan Provincial Science and Technology Department (no. 2012JY0125) and the Sichuan Provincial Bureau of Health (nos. 110259, 090538 and 130230).

\section{References}

1. Eluecque H, Gisquet H, Kitsiou C, et al: Pilomatrix carcinoma: A case report. J Clin Exp Dermatol Res 3: 1-3, 2012.

2. Sassmannshausen J and Chaffins M: Pilomatrix carcinoma: A report of a case arising from a previously excised pilomatrixoma and a review of the literature. J Am Acad Dermatol 44 (2 Suppl): 358-361, 2001.

3. Melancon JM, Tom WL, Lee RA, et al: Management of pilomatrix carcinoma: A case report of successful treatment with Mohs micrographic surgery and review of the literature. Dermatol Surg 37: 1798-1805, 2011.

4. Wang X, Ma JM and Wang N: Malignant pilomatricoma in the upper eyelid. BMJ Case Rep 2009: bcr07.2008.0410, 2009. 
5. Li X, Jiang $\mathrm{H}$ and Li A: Clinicopathological study on 15 cases of pilomatrix carcinoma. Zhonghua Bing Li Xue Za Zhi 26: 100-102, 1997 (In Chinese).

6. Karaaslan O, MelihCan M, Ozlem Karatas Silistreli A, Kaan Bedir Y and Caliskan G: Malignant pilomatrixoma arising on the previously irradiated face: Case report and literature review. J Cutan Med Surg 16: 341-343, 2012.

7. Joshi A, Sah SP, Agrawal CS, Jacob M and Agarwalla A: Pilomatrix carcinoma in a child. Acta Derm Venereol 79: 476-477, 1999.

8. Carbonaro V, Pietribiasi F and Penno A: Malignant pilomatrixoma of the face. Acta Otorhinolaryngol Ital 17: 444-447, 1997 (In Italian).

9. Hardisson D, Linares MD, Cuevas-Santos J and Contreras F: Pilomatrix carcinoma: A clinicopathologic study of six cases and review of the literature. Am J Dermatopathol 23: 394-401, 2001.

10. Aherne NJ, Fitzpatrick DA, Gibbons D, Collins CD and Armstrong JG: Recurrent malignant pilomatrixoma invading the cranial cavity: Improved local control with adjuvant radiation. J Med Imaging Radiat Oncol 53: 139-141, 2009.

11. Jani P, Chetty R and Ghazarian DM: An unusual composite pilomatrix carcinoma with intralesional melanocytes: Differential diagnosis, immunohistochemical evaluation, and review of the literature. Am J Dermatopathol 30: 174-177, 2008.
12. Kaddu S, Soyer HP, Wolf IH and Kerl H: Proliferating pilomatricoma. A histopathologic simulator of matrical carcinoma. J Cutan Pathol 24: 228-234, 1997.

13. De Gálvez-Aranda MV, Herrera-Ceballos E, Sánchez-Sánchez P, Bosch-García RJ and Matilla-Vicente A: Pilomatrix carcinoma with lymph node and pulmonary metastasis: Report of a case arising on the knee. Am J Dermatopathol 24: 139-143, 2002.

14. Cornejo KM and Deng A: Pilomatrix carcinoma: A case report and review of the literature. Am J Dermatopathol 35: 389-394, 2013.

15. Caubet-Biayna J, Ramos-Asensio R, Ortabe I and Mas M: Pilomatrix carcinoma of the face. J Oral Maxillofac Surg 57: 609-611, 1999.

16. Cozzi DA, d'Ambrosio G, Cirigliano E, et al: Giant pilomatricoma mimicking a malignant parotid mass. J Pediatr Surg 46: 1855-1858, 2011.

17. Sable D and Snow SN: Pilomatrix carcinoma of the back treated by mohs micrographic surgery. Dermatol Surg 30: 1174-1176,2004

18. Tselis N, Heyd R, Vogt HG and Zamboglou N: Pilomatrix carcinoma with lymph node and pulmonary metastases. Strahlenther Onko 182: 727-732, 2006.

19. Kim KU, Lee MK, Kim YS, et al: Pilomatrix carcinoma with lung and lymph node metastases. J Lung Cancer 7: 90-92, 2008. 